\title{
An Exact Ridge Matching Algorithm for Fingerprint Verification*
}

\author{
Jianjiang Feng ${ }^{1}$, Zhengyu Ouyang ${ }^{1}$, Fei Su${ }^{1}$, Anni Cai ${ }^{1}$ \\ ${ }^{1}$ Beijing University of Posts \& Telecommunications, Post Box 111, \\ Beijing 100876, P. R. China \\ fjianjiang@263.net, brian122@vip.sina.com, \{sufei, annicai\}@bupt.edu.cn
}

\begin{abstract}
Unlike traditional minutiae-based fingerprint matching approach, which establishes the minutiae correspondence between two fingerprints, in this paper a novel ridge-based fingerprint matching algorithm, which establishes the exact ridge correspondence between two fingerprints is proposed. The ridge correspondence is called 'exact' because correspondences between points on ridges are also established. The matching score is computed as the ratio of the length of matched ridges to that of all ridges. Experimental results on FVC2002 databases show that the ridge matching approach performs comparably to minutiae-based one, and an obvious improvement on matching performance can be obtained by combining the two matchers.
\end{abstract}

\section{Introduction}

Although fingerprint-based identification is one of the most mature and proven automatic personal identification techniques, there are still a lot of challenges that are often caused by the following reasons: (1) low quality fingerprint images; (2) small size fingerprint sensors. (3) non-linear distortion.

A large number of fingerprint matching algorithms have been proposed in the literature. According to [1], existing fingerprint matching approaches can be coarsely classified into three families: correlation-based matching [2, 3], minutiae-based matching $[4,5]$, and ridge feature-based matching [6, 7]. Correlation-based methods preserve most of the information in fingerprints, but are fragile to nonlinear distortion; Minutiae-based methods are flexible, but sensitive to noise; and Ridge feature-based methods are robust to noise, but seem to be less distinctive. Since each approach has its strengths and weaknesses, hybrid approaches are also proposed [8].

The fingerprint matching approach proposed in this paper does not belong to any of the three families. This approach tries to combine the advantages of three families of matching approaches. It has the potential ability to preserve most of the information in fingerprints like correlation-based methods, to be distinctive and flexible like minutiae-based methods, and to be robust to noise like ridge feature-based methods. This approach is based on ridges. Ridge image, also called thinned image or skeleton

\footnotetext{
* This work is supported by National Natural Science Foundation of China under grant 60472069 .
} 
image, is intermediate result in many fingerprint feature extraction algorithms. Although ridge-based fingerprint matching approaches have been neglected for many years, we think that ridge image is very suitable for matching due to its distinctiveness and robustness.

Although ridges have been used implicitly in the correlation-based matching algorithms [2] and explicitly in the alignment algorithm [5], only Isenor and Zaky [9] tried to identify fingerprint by matching ridges explicitly. However their algorithm only establishes the rough correspondences of ridges. That is to say, their algorithm determines ridge $a$ is matched to ridge $b$, but does not determine which portion of ridge $a$ is matched to which portion of ridge $b$. Since fingerprints are often partially overlapped and ridges can be broken or connected wrongly due to noise or imperfection of the feature extraction algorithm, such rough correspondence of ridges is not enough for representing the exact matching degree of two fingerprints from the same finger. Meanwhile, for different fingerprints with similar global ridge patterns, good rough correspondences of ridges might be established. Therefore, establishing only rough ridge correspondences is not enough to determine whether two fingerprints are from the same finger.

In this paper, a novel fingerprint matching algorithm is proposed, which establishes exact ridge correspondence between two fingerprints. In our approach, a ridge is represented by a list of points sampled equidistantly on the ridge, and the correspondences of such points are established. It is in this sense that the ridge correspondence is called 'exact'. The ridge matching algorithm works as follows. First, a pair of ridges that are most similar is found. Then this pair of ridges is used as the base pair, and the ridges next to them are compared. Consequently the new matched ridges are used as the base pair, and the ridges next to them are compared, and so on. This recursive procedure proceeds until no more ridges can be matched. At last, a matching score is computed based on the length of matched ridges. Since the first base pair of ridges may not be the true matched pair, multiple pairs of ridges can be chosen as the initial base pairs and the above recursive matching procedure can be performed for multiple times. Finally the maximum score of the multiple procedures is used as the final matching score of the two fingerprints. Experiment results on FVC2002 databases [10] show that the proposed ridge-based matching approach performs comparably to the minutiae-based one, and an obvious improvement on matching performance is obtained by combining the two matchers.

In the next section, we describe the ridge matching algorithm in detail. The experimental results and analysis are given in section 3 . Finally in section 4, summary is presented and future directions are suggested.

\section{Ridge matching}

Given two grayscale fingerprint images, $I_{1}$ and $I_{2}$, we obtain their ridge images (thinned image), $T_{1}$ and $T_{2}$, and minutiae sets, $M_{1}$ and $M_{2}$, using an algorithm developed in our laboratory, which is similar to the algorithm described in [5]. Then our ridge matching algorithm accepts as input the two ridge images and the two minutiae sets, and outputs a score that indicates the degree of similarity between the two fin- 
gerprints. In 2.1, the representation of ridges is described. In 2.2, how to compare two aligned ridges is explained. In 2.3, how to find a set of initial base pairs of ridges is described. Given an initial pair of ridges, how to match all the remaining ridges, the most important part of the matching process, is presented in 2.4. In 2.5, the concept of relational distance is adopted to prevent false matches. Finally in 2.6, the computation of the matching score is described.

\subsection{Representation of Ridges}

Due to noise in fingerprint images and imperfection of the feature extraction methods, there are complex structures, such as loops and bridges, in the ridge images. In order to make the ridge structure simple and consequently the following matching algorithm easier, a pre-processing step is performed: (1) Closed ridges are disconnected at an arbitrary point; (2) Ridges associated with bifurcations are split into three ridges; (3) Short ridges are removed. In the matching stage, it is unnecessary and time-consuming to examine every point on a ridge. Therefore, each ridge is sampled equidistantly and is represented by the list of sampled points.

\subsection{Match Aligned Ridges}

Given two aligned ridges, $\left\{a_{i}\right\}_{i=1}^{M}$ and $\left\{b_{j}\right\}_{j=1}^{N}$. The task of matching them is to find the longest continuous matched point strings. Two points are matched if their Euclidean distance is less than a threshold. We created an array $T(i, j), i=1, \cdots, M ; j=1, \cdots, N$. $T(i, j)=1$ means $a_{i}$ and $b_{j}$ are matched. $T(i, j)=0$ means $a_{i}$ and $b_{j}$ are unmatched.

A list of index pairs $\left\{\left(P_{l}, Q_{l}\right)\right\}_{l=1}^{K}$ will be found, where $P_{l}, Q_{l}$ are indices of $\left\{a_{i}\right\}_{i=1}^{M}$ and $\left\{b_{j}\right\}_{j=1}^{N}$ respectively, and $P_{l}, Q_{l}$ should satisfy the following conditions: (1) $T\left(P_{l}, Q_{l}\right)=1$; (2) $P_{l}, Q_{l}$ monotonously increase or decrease respectively; (3)

$$
\left|P_{l}-P_{l-1}\right| \leq 2,\left|Q_{l}-Q_{l-1}\right| \leq 2 ; l=2, \ldots, K .
$$

The last condition means that to make the algorithm more robust to noise, slight discontinuity in a matched point string is allowed. Such point strings can be found in $T$ using dynamic programming technique [11]. If the longest list consists of more than $K_{\text {th }}$ (i.e., $K_{\mathrm{th}}=3$ ) pairs of points, the correspondence between matched points will be recorded.

\subsection{Find the Initial Base Pairs of Ridges}

This step is to find a set of $N$ initial matched ridge pairs $\left\{\left(r_{1, i}, r_{2, j}\right) \mid r_{1, i} \in R_{1}, r_{2, j} \in R_{2}\right\}$ from two ridge sets $R_{1}$ and $R_{2}$. Since the pose transformation between the two fingerprints is unknown in advance, we restrict our search space to ridges associated with minutiae. We use the approach proposed in [5] to measure the similarity degree between any two ridges. The top $N$ ridge pairs are selected as the initial matched ridge pairs. 


\subsection{Match All Ridges}

Given an initial pair of matched ridges, the ridges next to them can be aligned and paired. Then the ridges next to the newly matched ridges can be aligned and paired, and so on. Such procedure can be implemented as a recursive function. The pseudo-codes of the recursive function MatchNearbyRidges is given below. The input of the function is a pair of matched ridges $b r_{1}$ and $b r_{2}$, which will be used as the base ridge pair. When this function is called for the first time, the base ridge pair is one of the initial pairs of matched ridges. The function works as follows. First, the local pose transformation between $b r_{1}$ and $b r_{2}$ is computed. Then, for each pair of matched points in the base ridge pair and at each side of the base ridge, search for the possible matched ridges. For each pair of the possible matched ridges, align and match them using the approach described in subsection 2.2. Finally, each pair of ridges that is just matched during this call is used as the new base ridge pair to call MatchNearbyRidges in turn.

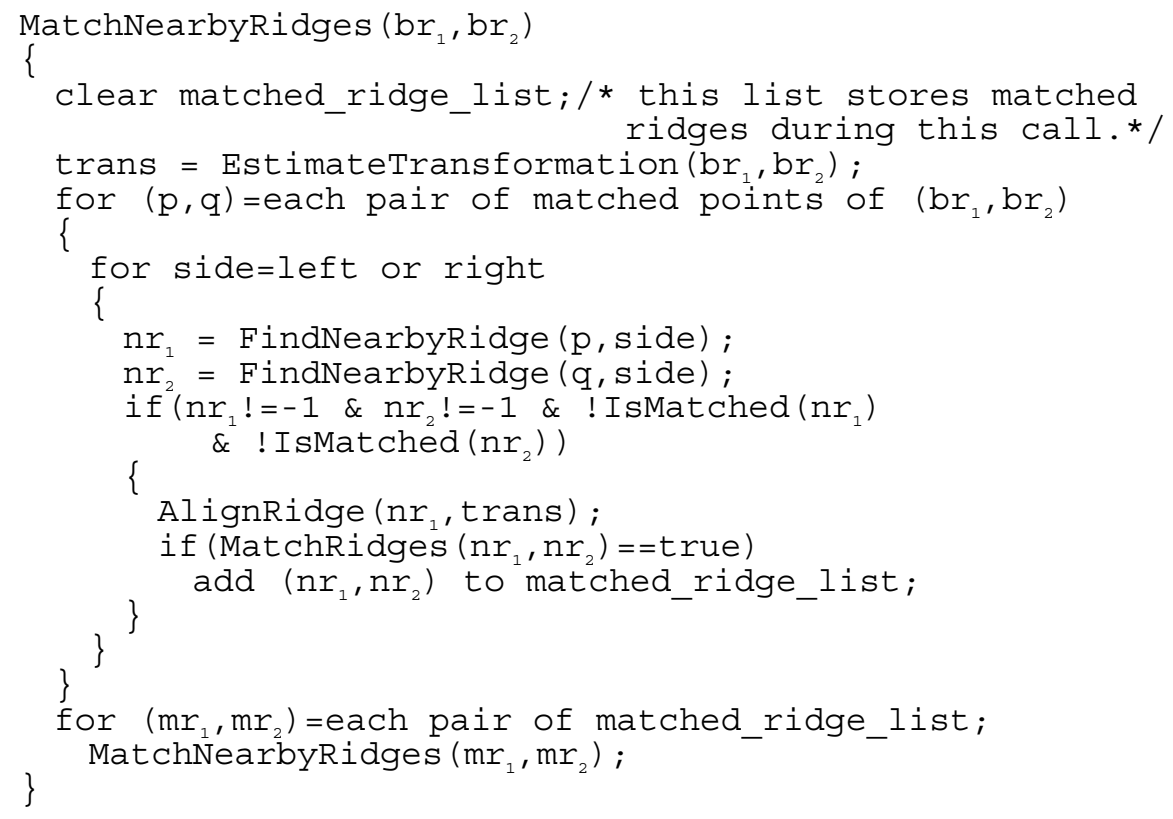

The result of comparing two aligned ridges can be completely unmatched, completely matched or partly matched. Due to noise, nonlinear deformation, partial overlap, and imperfection of the feature extraction algorithm, the corresponding ridges between two fingerprints from the same finger are not necessarily completely matched. Therefore, in the case of partly matched, unmatched parts of the ridges will be left over as new ridges that will be matched later. 


\subsection{Consistency Constraint}

As described in the previous section, in order to deal with in-class variations in ridge structures, a ridge can be split into several short ridges. As a result, there are dangers that ridges from different fingers may match well with each other due to multiple splits. We adopted the concept of relational distance defined by Haralick and Shapiro [12] to prevent such cases.

Let $\mathrm{MR}_{1}=\left\{m r_{1, i}\right\}_{i=1, \ldots, N}$ and $\mathrm{MR}_{2}=\left\{m r_{2, i}\right\}_{i=1, \ldots, N}$ denote two sets of ridges, where ridge $m r_{1, i}$ is matched to ridge $m r_{2, i}$. We will define adjacency relation for matched ridges in the following fashion. Ridge $i$ and ridge $j$ have adjacency relation if they are from the same ridge and directly connected. Adjacency relation also contains the information whether head or tail of ridges is adjacent. The head and tail of a ridge can be specified arbitrarily, but for matched ridges, they should be consistent. Such information is recorded when a pair of ridges is found to be partly matched and ridges are split into multiple ridges. Let $s=(i, j, e i, e j)$ denote a relation, which means ei of ridge $i$ and $e j$ of ridge $j$ are adjacent, where $e i$, ej can be head or tail. Let $S_{1}=\left\{s_{1, i}\right\}_{i=1, \ldots, N_{1}}$ and $\mathrm{S}_{2}=\left\{s_{2, i}\right\}_{i=1, \ldots, N_{2}}$ denote all relations over $\mathrm{MR}_{1}$ and $\mathrm{MR}_{2}$, respectively. We say relation $s_{1}=\left(i_{1}, j_{1}, e i_{1}, e j_{1}\right)$ and relation $s_{2}=\left(i_{2}, j_{2}, e i_{2}, e j_{2}\right)$ are matched if one of the following conditions is satisfied: (1) Ridge $i_{1}$ is matched to $i_{2}$, and ridge $j_{1}$ is matched to $j_{2}$, and $e i_{1}=e i_{2}$, and $e j_{1}=e j_{2} ;(2)$ Ridge $i_{1}$ is matched to $j_{2}$, and ridge $j_{1}$ is matched to $i_{2}$, and $e i_{1}=e j_{2}$, and $e j_{1}=e i_{2}$.

Using the above definitions, we can compute the relational distance as follows. At first, set the relational distance to 0 . For each relation in $S_{1}$, if no matched relation can be found in $S_{2}$, relational distance is increased by 1 . Then for each relation in $\mathrm{S}_{2}$, if no matched relation can be found in $\mathrm{S}_{1}$, relational distance is increased by 1 . We can reasonably expect that the relational distance of a genuine match should be small, and it is 0 when ridge matches are completely consistent. If the relational distance is greater than a threshold, the matching score for this matching procedure is set to 0 .

\subsection{Matching Score}

As two fingerprints may share a small overlapped region, in order to evaluate the similarity degree of them, we should consider only the matching status in the overlapped region. For each of the two fingerprints, compute the convex hull of ridge images. The overlapped region is the intersection of two convex hulls that are aligned according to the initial pairs of ridges. The matching score $\operatorname{score}_{i}\left(0 \leq \operatorname{score}_{i} \leq 1\right)$ of the ith matching procedure can be computed according to the following formula:

$$
\text { score }_{i}=\frac{N_{i m}{ }^{2}}{N_{i 1} \cdot N_{i 2}} \text {. }
$$


where $N_{i 1}$ and $N_{i 2}$ is the number of ridge points of two fingerprints in overlapped region, and $N_{i m}$ is the number of matched sampled points. Then the final matching score is equal to the maximum of score $_{i}$.

\section{Experimental Results}

Experiments have been conducted on DB1_A of FVC2002. This databases consists of 800 fingerprint images from 100 fingers (eight impressions per finger). The performance evaluation protocol used in FVC2002 [10] has been adopted. For genuine match, each impression of each finger is compared with other impressions of the same finger. The number of matches is $C_{8}^{2} \times 100=2800$. For impostor match, the first impression of each finger is compared with the first impression of other fingers. The number of matches is $C_{100}^{2}=4950$.

Two examples, one for genuine match and another for impostor match, are given in Fig. 1. From the figure, we can see that most of the ridges in the overlapped region of two fingerprints from the same finger are matched, while most of the ridges in the overlapped region of two fingerprints from different fingers are unmatched. For examples shown in this figure, the matching score of genuine match is 0.95 and the matching score of impostor match is 0.11 , which are obviously easy to be distinguished from each other.

The algorithm presented in this paper is compared with a minutiae-based matching algorithm previously developed in our lab. The ROC (Receiver Operating Characteristic) curves of these two methods are plotted in Fig. 2. The EERs of the ridge-based one and the minutiae-based one are $1.2 \%$ and $1.8 \%$, respectively. In terms of EER (Equal Error Rate), the performance of ridge matching approach is slightly better than that of minutia matching one. In terms of ROC, however, it is hard to determine which one is better. In the region of low threshold, the performance of ridge matching approach is better than that of minutia matching one. In the region of high threshold, the result turns out contrary. Superiority in the region of low threshold means that the ridge matching approach is good at handling difficult genuine matches. This proves that, due to amount of information carried by ridges, the ridge matching approach is relatively robust to noise, small overlap area and nonlinear deformations. The Inferiority in the region of high threshold means that the current ridge matching approach behaves not well when handling difficult impostor matches. This should be caused by the following factors: the ridge images of different fingerprints of the same category can be similar, and local ridge images of different fingerprints can be similar too. However, we do not think this means distinctiveness of the ridge matching approach is lower. There is plenty of room for improvement of the current algorithm.

Both intuition and analysis on ROC curves remind us that ridges are complementary to minutiae and the combination of the two approaches could provide better performance. Therefore, a classifier combination with sum of matching scores given by the two matchers is tested on the same databases, using the same performance evaluation protocol. The EER is 1.0\%. The ROC curves are also plotted in Fig. 2. From the figure, we can see that the ROC curve of the combiner is better than any single matcher in the whole threshold region. 
The above experiments were conducted on a PC with P4 2.2 processor, running Windows 2000. The matching times of the ridge-based one and the minutiae-based one are $63 \mathrm{~ms}$ and $9 \mathrm{~ms}$, respectively. Since the number of ridge points is approximately 20 times larger than that of minutiae, this fact is acceptable.

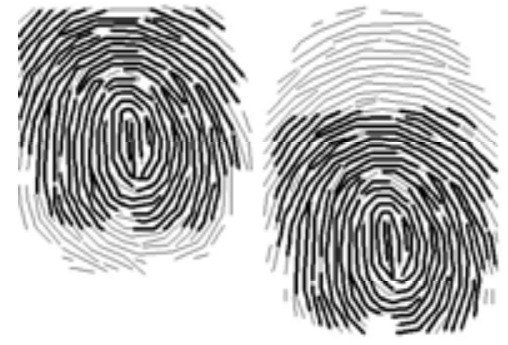

(a)

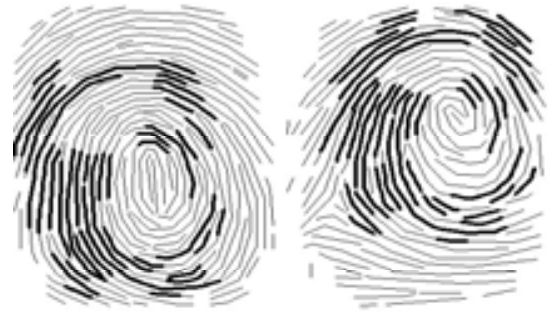

(b)

Fig. 1. (a) Match result for fingerprints from same finger; (b) Match result for fingerprints from different fingers. Thick lines represent matched ridges and thin lines represent unmatched ridges

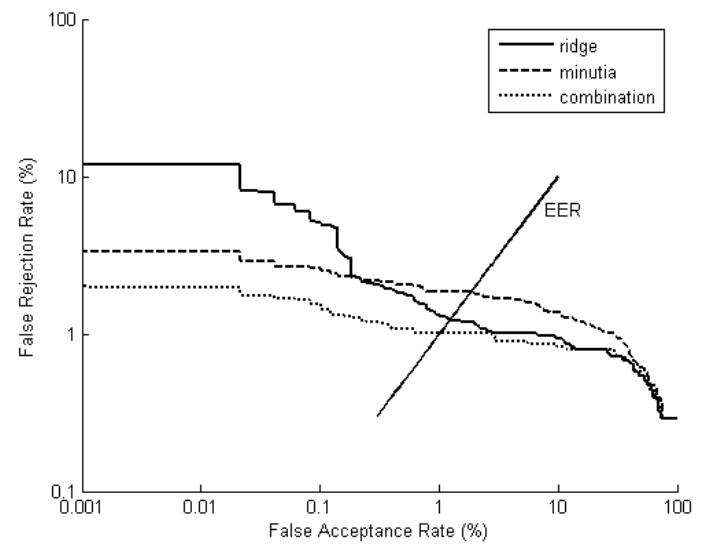

Fig. 2. ROC curves of ridge-based (solid line), minutiae-based (dashed line), combination algorithm (dot line) on DB1_A of FVC2002

\section{Summary and Future Work}

We have proposed a ridge-based fingerprint matching algorithm. The basic idea is to establish the exact ridge correspondence between two fingerprints and compute matching score based on the length of matched ridges. The algorithm incrementally matches ridges using a recursive function. To allow for in-class variations in ridge structures, ridges can be split into small ridges. To prevent impostor matches due to 
ridge splitting, consistency constraints are imposed. Experiments on FVC2002 databases show that the ridge-based approach performs comparably to the minutiae-based one and the matching performance can be further improved by a combination of the two complementary matchers.

There are some defects in current ridge matching algorithm. For genuine matches, searching for local optimal matches of individual ridges does not necessarily lead to a global optimal match of the whole ridge patterns; moreover, the matching procedure can be stopped at seriously damaged regions of the ridge images. For impostor matches, the local ridge relations exploited currently are too weak. Stronger consistent constraints should be introduced in our work in future. Furthermore, we notice that that in a ridge image, some ridges are more important or reliable than others. Therefore, it will be beneficial to assign a weight to each ridge to indicate its importance and to incorporate this information into the matching algorithm. Finally, the computational complexity of the algorithm should also be decreased.

\section{References}

1. Maltoni, D., Maio, D., Jain, A.K., Prabhakar, S.: Handbook of Fingerprint Recognition. Springer-Verlag, Berlin Heidelberg New York (1996)

2. Wilson, C., Watson, C., Paek, E.: Effect of Resolution and Image Quality on Combined Optical and Neural Network Fingerprint Matching. Pattern Recognition 33 (2) (2000) 317-331

3. KovQacs-Vajna, Z.M.: A Fingerprint Verification System Based on Triangular Matching and Dynamic Time Warping. IEEE Trans. Pattern Anal. Mach. Intell. 22 (11) (2000) 1266-1276

4. Ratha, N.K., Karu, K., Chen, S., Jain, A.K.: A Real-time Matching System for Large Fingerprint Databases. IEEE Trans. Pattern Anal. Mach. Intell. 18 (8) (1996) 799-813

5. Jain, A.K., Hong, L., Bolle, R.: On-line Fingerprint Verification. IEEE Trans. Pattern Anal. Mach. Intell. 19 (4) (1997) 302-313

6. Jain, A.K., Prabhakar, S., Hong, L., Pankanti, S.: Filterbank-based Fingerprint Matching. IEEE Trans. Image Processing 9 (5) (2000) 846-859

7. Coetzee, L., Botha, E.C.: Fingerprint Recognition in Low Quality Images. Pattern Recognition 26 (10) (1993) 1441-1460

8. Ross, A., Jain, A.K. Reisman, J.: A Hybrid Fingerprint Matcher. Pattern Recognition 36 (7) (2003) 1661-1673

9. Isenor, D.K., Zaky, S.G.: Fingerprint Identification Using Graph Matching. Pattern Recognition 19 (2) (1986) 113-122

10. Maio, D., Maltoni, D., Cappelli, R., Wayman, J.L., Jain, A.K.: FVC2002: Second Fingerprint Verification Competition. In: Proceedings of the International Conference on Pattern Recognition (ICPR), Quebec City, Canada (2002) 744-747

11. Cormen, T.H., Leiserson, C.E., Rivest, R.L.: Introduction to Algorithms. McGraw-Hill, New York (1990)

12. Haralick, R., Shapiro, L.: The Consistent Labeling Problem II. IEEE Trans. Pattern Anal. Mach. Intell. 2 (1980) 193-203 